\title{
Background-Free 3D Nanometric Localization and Sub-nm Asymmetry Detection of Single Plasmonic Nanoparticles by Four-Wave Mixing Interferometry with Optical Vortices
}

\author{
George Zoriniants, ${ }^{1}$ Francesco Masia, ${ }^{2}$ Naya Giannakopoulou, ${ }^{1}$ Wolfgang Langbein, ${ }^{2, *}$ and Paola Borri ${ }^{1, \dagger}$ \\ ${ }^{1}$ Cardiff University School of Biosciences, Museum Avenue, Cardiff CF10 3AX, United Kingdom \\ ${ }^{2}$ Cardiff University School of Physics and Astronomy, The Parade, Cardiff CF24 3AA, United Kingdom \\ (Received 2 June 2017; revised manuscript received 27 August 2017; published 27 October 2017)
}

Single nanoparticle tracking using optical microscopy is a powerful technique with many applications in biology, chemistry, and material sciences. Despite significant advances, localizing objects with nanometric position precision in a scattering environment remains challenging. Applied methods to achieve contrast are dominantly fluorescence based, with fundamental limits in the emitted photon fluxes arising from the excited-state lifetime as well as photobleaching. Here, we show a new four-wave-mixing interferometry technique, whereby the position of a single nonfluorescing gold nanoparticle of 25-nm radius is determined with $16 \mathrm{~nm}$ precision in plane and $3 \mathrm{~nm}$ axially from rapid single-point measurements at 1 -ms acquisition time by exploiting optical vortices. The precision in plane is consistent with the photon shot-noise, while axially it is limited by the nano-positioning sample stage, with an estimated photon shot-noise limit of $0.5 \mathrm{~nm}$. The detection is background-free even inside biological cells. The technique is also uniquely sensitive to particle asymmetries of only $0.5 \%$ ellipticity, corresponding to a single atomic layer of gold, as well as particle orientation. This method opens new ways of unraveling single-particle trafficking within complex 3D architectures.

DOI: 10.1103/PhysRevX.7.041022

\section{INTRODUCTION}

Many complex dynamics and interactions in physical, chemical, and biological processes occur at the nanometer scale within submilliseconds. A powerful way to study these interactions is based on tracking the trajectory of a single nanoscale object using optical microscopy (for a recent review, see Ref. [1]).

To achieve sufficient contrast and specificity against backgrounds in heterogeneous environments (such as, for example, biological cells), the methods often rely on tracking fluorescing molecules. However, fluorophores are single-quantum emitters and are thus only capable of emitting a certain maximum number of photons per unit time because of the finite duration of their excited-state lifetime; moreover, they are prone to photobleaching and associated phototoxicity. As a result, the spatial position precision and trajectory time window of single-particle tracking by means of fluorescent reporters is typically restricted to $20-50 \mathrm{~nm}$ and a few seconds, respectively $[1,2]$.

\footnotetext{
langbeinww@cardiff.ac.uk

tborrip@cardiff.ac.uk
}

Published by the American Physical Society under the terms of the Creative Commons Attribution 4.0 International license. Further distribution of this work must maintain attribution to the author(s) and the published article's title, journal citation, and DOI.
Subject Areas: Interdisciplinary Physics, Nanophysics, Optics

Alternatively to using fluorescent emitters, singleparticle tracking can be implemented with nonfluorescing nanoparticles (NPs) that have strong optical responses, such as second-harmonic (SH) active nanocrystals [3] and plasmonic (usually metallic) NPs [4]. However, the practical application of $\mathrm{SH}$ active nanocrystals for rapid tracking appears limited to fairly large NP sizes $(\geq 100 \mathrm{~nm}$ diameter) to achieve enough signal-to-noise ratio and overcome endogenous background in biological cells [5]. These large NPs might alter the intrinsic motion of the molecules of interest to which they are attached and/or be incompatible with physiological cellular uptakes. Conversely, tracking of small metallic NPs has been shown, as they are selectively detected via their strong scattering and absorption at the localized surface plasmon resonance (LSPR) [4]. This detection is photostable, and the achievable photon fluxes are governed by the incident photon fluxes and the NP optical extinction cross section. Owing to the large number of photons making up the image of an individual particle, its center position coordinates can be determined with high precision, much better than the diffraction-limited point spread function (PSF) of a microscope objective $[1,2]$. This has been shown for metallic NPs using wide-field imaging techniques such as bright-field microscopy [6], dark-field microscopy [7], differential interference contrast [8], and interferometric scattering microscopy [9]. These techniques, however, are not background-free and either use large NPs to distinguish them from endogenous scattering and phase contrast in 
heterogeneous samples or work in optically clear environments. A more selective technique uses photothermal imaging where the image contrast originates from the refractive index change in the region surrounding the nanoparticle due to local heating following light absorption. This is a focused-beam scanning technique, and it has been used to track single 5-nm diameter gold NPs in two dimensions (however, not 3D) with an estimated 20-nm precision, using a beam triangulation method at a $30-\mathrm{Hz}$ tracking rate [10]. More recently, single 40-nm-diameter gold NPs have been imaged in 3D by photothermal optical coherence microscopy with $0.5-\mu \mathrm{m}$ lateral and $2-\mu \mathrm{m}$ axial resolution, but tracking was not reported [11]. By detecting the nanoparticle only indirectly via the photothermal index change generated in its surrounding, this method is not free from backgrounds either. In fact, photothermal contrast has been shown in the absence of NPs due to endogeneous absorption in cells [12]. Generally, for all these techniques, rapid tracking of small NPs in 3D with high precision in a scattering environment is challenging.

Beyond translational motion, many chemical or biological processes also involve rotational dynamics. To that end, there has been growing interest in developing optical methods able to measure the geometrical anisotropy of single NPs, as a probe for tracking rotations [13,14]. Generally, optical techniques aiming at characterizing the anisotropy of single NPs employ polarization-resolved excitation and/or detection, combined with high numerical aperture (NA) objectives for high spatial resolution. Besides signal-to-noise and background considerations of the various optical methods (absorption-based, scattering-based, fluorescence-based $[4,15,16])$, the polarization mixing induced by such high NA objectives $[17,18]$ is often not accounted for in the analysis and can limit the sensitivity to particle anisotropies. For plasmonic particles, sensitivity to ellipticity in the 5\% range has been shown using polarization-resolved dark field [19] and extinction microscopy [20,21], although, in practice, most experiments using techniques such as polarization-resolved spatial modulation microscopy [22], photothermal imaging [23], two-photon luminescence [24], and SH generation [25] are carried out with largely anisotropic nanorods of aspect ratios greater than 2 .

We have shown that four-wave mixing (FWM), triply resonant to the LSPR, is a very selective, high-contrast photostable method to detect single small gold NPs [26,27]. It is a third-order nonlinearity that originates from the change in the NP dielectric constant induced by the resonant absorption of a pump pulse and subsequent formation of a nonequilibrium hot electron gas in the metal [27]. It is therefore very specific to metallic NPs, which are imaged background-free even in highly scattering and fluorescing environments [26]. In this work, we show theoretically and experimentally a new FWM detection modality that can determine the position of a single gold NP in the 15-30-nm radius range with shot-noiselimited precision better than $20 \mathrm{~nm}$ in plane and $1 \mathrm{~nm}$ axially from scanless single-point background-free acquisition on a $1 \mathrm{~ms}$ time scale, by exploiting optical vortices of tightly focused light. The technique is also uniquely sensitive to particle asymmetries down to $0.5 \%$ ellipticity, corresponding to a single atomic layer of gold.

\section{FOUR-WAVE-MIXING INTERFEROMETRY TECHNIQUE}

A sketch of the FWM technique is shown in Fig. 1(a). The key developments in this new design compared to previous works are the epicollection (reflection) geometry and the dual-polarization heterodyne detection scheme. We use a train of femtosecond optical pulses with repetition rate $\nu_{L}$, which is split into three beams, all having the same center optical frequency $\nu_{0}$, resulting in a triply degenerate FWM scheme. One beam acts as a pump and excites the NP at the LSPR with an intensity that is temporally modulated with close to unity contrast by an acousto-optic modulator $\left(\mathrm{AOM}_{1}\right)$ driven at carrier frequency $\nu_{1}$ with a square-waveamplitude modulation of frequency $\nu_{m}$. The change in the NP optical properties induced by this excitation is resonantly probed by a second pulse at an adjustable delay time $\tau$ after the pump pulse. Pump and probe pulses of fields $\mathbf{E}_{1}$ and $\mathbf{E}_{2}$, respectively, are recombined into the same spatial mode and focused onto the sample by a high NA microscope objective. The sample can be positioned and moved with respect to the focal volume of the objective by scanning an $x y z$ sample stage with nanometric position precision. A FWM field $\mathbf{E}_{\mathrm{FWM}}$ (proportional to $\mathbf{E}_{1} \mathbf{E}_{1}^{*} \mathbf{E}_{2}$ ) is collected, together with the probe in reflection (epidirection) by the same objective, transmitted by the beam splitter $\left(\mathrm{BS}_{1}\right)$ used to couple the incident beams onto the microscope, and recombined in a second beam splitter $\left(\mathrm{BS}_{2}\right)$ with a reference pulse field $\left(\mathbf{E}_{R}\right)$ of adjustable delay. The resulting interference is detected by two pairs of balanced photodiodes. A heterodyne scheme discriminates the FWM field from pump and probe pulses and detects the amplitude and phase of the field. In this scheme, the probe optical frequency is slightly upshifted via a second $\mathrm{AOM}\left(\mathrm{AOM}_{2}\right)$, driven with a constant amplitude at a radio frequency of $\nu_{2}$, and the interference of the FWM with the unshifted reference field is detected. As a result of the amplitude modulation of the pump at $\nu_{m}$ and the frequency shift of the probe at $\nu_{2}$, this interference gives rise to a beat note at $\nu_{2}$, with two sidebands at $\nu_{2} \pm \nu_{m}$, and replica separated by the repetition rate $\nu_{L}$ of the pulse train. A multichannel lock-in amplifier enables the simultaneous detection of the carrier at $\nu_{2}-\nu_{L}$ and the sidebands at $\nu_{2} \pm \nu_{m}-\nu_{L}$. These frequencies (see the Appendix) are around $2 \mathrm{MHz}$, and the separation of the sidebands is $400 \mathrm{kHz}$, much higher than the lock-in amplifier detection bandwidth (inverse of twice the pixel dwell time) in the $1 \mathrm{kHz}$ range; i.e., the detected signals have no significant cross talk. Via the 
in-phase ( $\mathrm{Re})$ and in-quadrature $(\mathrm{Im})$ components for each detected frequency, the amplitude and phase of the probe field reflected by the sample $\mathbf{E}_{2 \mathrm{r}}$ (detected at $\nu_{2}-\nu_{\mathrm{L}}$ ) and of the epidetected FWM field $\mathbf{E}_{\mathrm{FWM}}\left(\right.$ at $\nu_{2} \pm \nu_{m}-\nu_{L}$ ) are measured [see sketches in Fig. 1(a)].

A key point of the technique is the use of a dualpolarization balanced detection. First, probe and pump beams, linearly polarized horizontally $(H)$ and vertically $(V)$, respectively, in the laboratory system, are transformed into cross-circularly polarized beams at the sample by a combination of $\lambda / 4$ and $\lambda / 2$ wave plates. The reflected probe and FWM fields collected by the same microscope objective travel backwards through the same wave plates, such that the probe reflected by a planar surface returns $V$ polarized in the laboratory system. The reference beam is polarized at $45^{\circ}$ (using a polarizer) prior to recombining with the epidetected signal via the nonpolarizing beam splitter $\mathrm{BS}_{2}$. A Wollaston prism vertically separates $\mathrm{H}$ and $\mathrm{V}$ polarizations for each arm of the interferometer after $\mathrm{BS}_{2}$. Two pairs of balanced photodiodes then provide polarization-resolved detection, the bottom (top) pair detecting the current difference (for common-mode noise rejection) of the $V(H)$ polarized interferometer arms. In turn, this corresponds to detecting the co- and cross-circularly

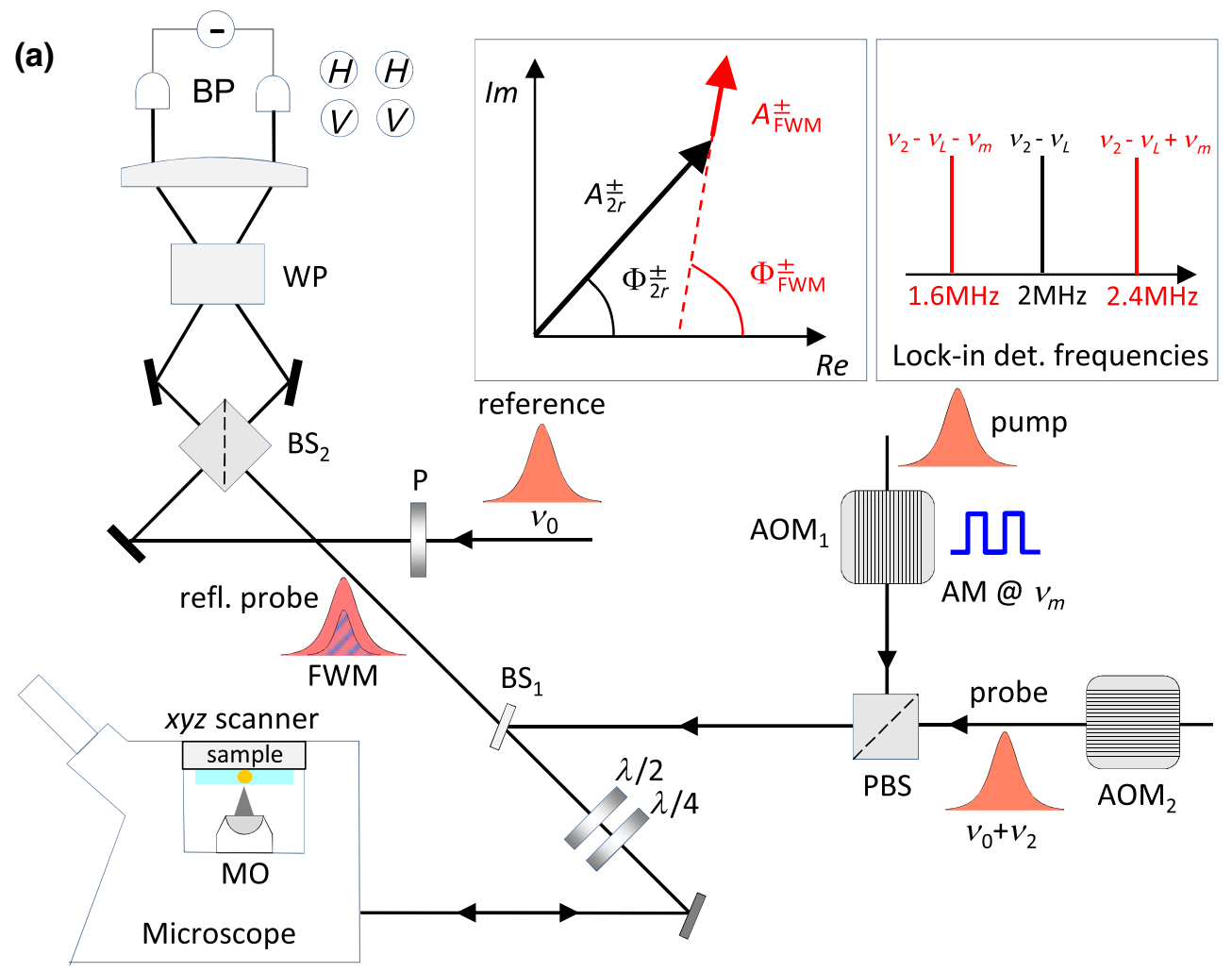

(b)
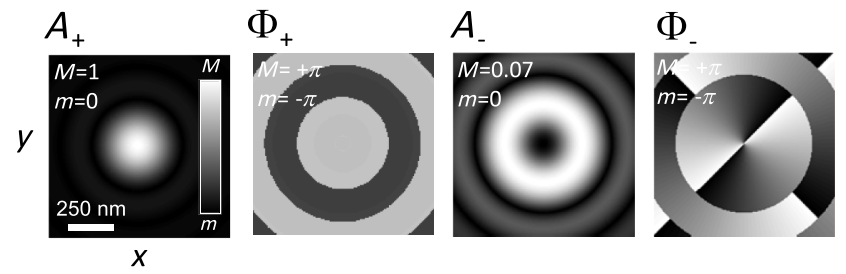

FIG. 1. Four-wave-mixing interferometry epidetected dual-polarization resolved. (a) Sketch of pump pulses that are amplitude modulated (AM) at $\nu_{m}$ and probe pulses that are frequency shifted by $\nu_{2}$, using acousto-optical modulators (AOMs). Pulses are coupled into an inverted microscope equipped with a high NA microscope objective (MO). Pump and probe beams are adjusted to be circularly polarized at the sample by $\lambda / 4$ and $\lambda / 2$ wave plates. Circular polarizations are transformed into horizontal $(H)$ and vertical $(V)$ linear polarization by the same wave plates, and both components are simultaneously detected through their interference with a frequencyunshifted reference linearly polarized at $45^{\circ}$. BP: Balanced photodiodes. WP: Wollaston prism deflecting beam out of drawing plane. $(P)$ BS: (Polarizing) Beam splitter. $P$ : Polarizer. Inset: Amplitude $(A)$ and phase $(\Phi)$ of the reflected probe and FWM field, measured by a multichannel lock-in, where $+(-)$ refers to the co-(cross-)polarized component relative to the incident circularly polarized probe. (b) Calculated field distribution in the focal plane of a $1.45 \mathrm{NA}$ objective for an incident field left circularly polarized. Here, $A_{+}\left(A_{-}\right)$is the co-(cross-)polarized amplitude, and $\Phi_{+}\left(\Phi_{-}\right)$is the corresponding phase. 

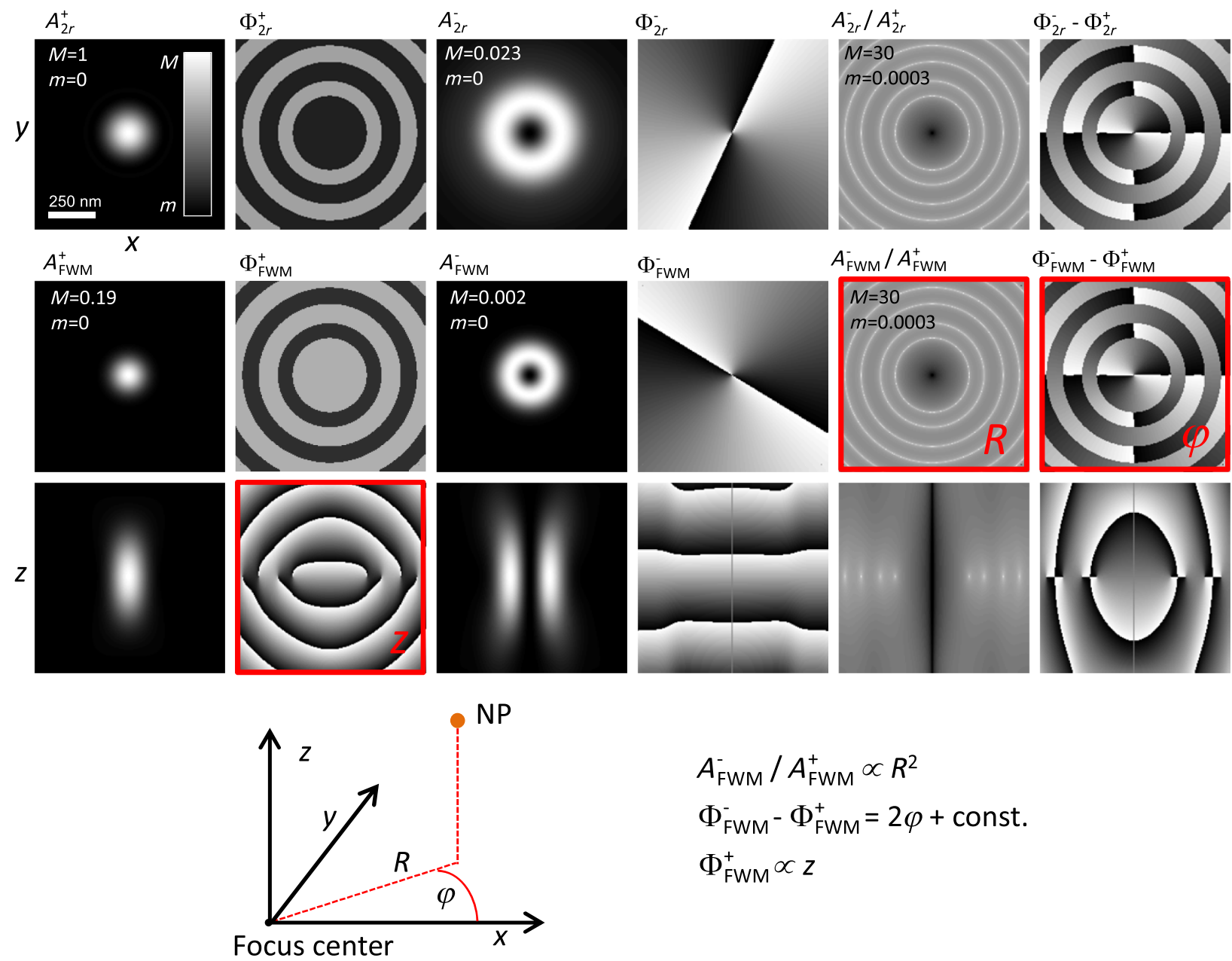

$$
\begin{aligned}
& A_{\mathrm{FWM}}^{-} / A_{\mathrm{FWM}}^{+} \propto R^{2} \\
& \Phi_{\mathrm{FWM}}^{-}-\Phi_{\mathrm{FWM}}^{+}=2 \varphi+\text { const. } \\
& \Phi_{\mathrm{FWM}}^{+} \propto z
\end{aligned}
$$

FIG. 2. Nanometric localization using optical vortices. Calculated amplitude (phase) components of the reflected probe field and FWM field, $A_{2 r}^{ \pm}$and $A_{\mathrm{FWM}}^{ \pm}\left(\Phi_{2 r}^{ \pm}\right.$and $\left.\Phi_{\mathrm{FWM}}^{ \pm}\right)$, respectively, as a function of particle position in the sample focal plane $(x, y)$, and in a section along the axial direction $(x, z)$ through the focus, where + refers to the co-polarized component and - to the cross-polarized component relative to the left-circularly polarized incident probe. The calculation assumes a perfectly spherical gold NP in the dipole approximation. The inset shows a sketch of how the amplitude and phase of the FWM field ratio and the phase of the co-polarized FWM field can be used to locate, in 3D, the spatial position of the NP relative to the focus center. The linear grey scale is from $-\pi$ to $\pi$ for all phases, and from $m$ to $M$ for field amplitudes, as indicated. The amplitude ratios of reflected probe and FWM are shown on a logarithmic grey scale over 5 orders of magnitude.

polarized components of $\mathbf{E}_{2 \mathrm{r}}$ and $\mathbf{E}_{\mathrm{FWM}}$ relative to the incident circularly polarized probe, having amplitudes (phases) indicated as $A_{2 \mathrm{r}}^{ \pm}$and $A_{\mathrm{FWM}}^{ \pm}\left(\Phi_{2 \mathrm{r}}^{ \pm}\right.$and $\left.\Phi_{\mathrm{FWM}}^{ \pm}\right)$in the sketch in Fig. 1(a), where $+(-)$ refers to the co-(cross-) polarized component.

\section{CONCEPT OF NANOMETRIC LOCALIZATION USING OPTICAL VORTICES}

To conceptually elucidate how the nanometric position precision arises from this dual-polarization-resolved FWM interferometry detection scheme, we numerically simulated the field distribution in the focal region of a $1.45 \mathrm{NA}$ objective. The simulation parameters (wavelength, coverslip thickness, medium refractive index, back-objective filling factor) were chosen to match the actual experimental conditions (see the Appendix). The amplitude and phase components of the field in the focal plane are shown in Fig. 1(b) for a left circularly $\left(\sigma^{+}\right)$polarized input field. Because of the high NA of the objective and the vectorial nature of the field [17], there is a significant cross-circularly polarized component that forms an optical vortex of topological charge $l=2$; i.e., it has an amplitude $\left(A_{-}\right)$, which is zero in the focus center and radially symmetric nonzero away from the center, and a phase $\left(\Phi_{-}\right)$changing with twice the in-plane polar angle. A pointlike gold NP displaced from the focus center experiences this field distribution and will in turn emit a field with an amplitude and a phase directly related to the NP radial and angular position. While this is the case for both reflected and FWM 
fields, only the FWM signal is background-free and hence suited for tracking the NP in heterogeneous environments. In the following, we discuss the FWM field emitted from such NP and the corresponding coordinate retrieval.

We calculate the FWM field starting from the polarization of the NP at position $\mathbf{r}$ induced by the probe field, described by $\mathbf{p}(\mathbf{r})=\epsilon_{0} \epsilon_{m} \hat{\alpha} \mathbf{E}_{2}(\mathbf{r})$, where $\epsilon_{0}$ is the vacuum permittivity, $\epsilon_{m}$ is the dielectric constant of the medium surrounding the NP (glass and silicon oil in the experiment), and $\hat{\alpha}$ is the particle polarizability tensor. We calculate $\hat{\alpha}$ in the dipole approximation, valid for particle sizes much smaller than the wavelength of light (Rayleigh regime). To take into account nonsphericity of real particles, which will be relevant in the experiments as shown later, we adopted the model of a metallic ellipsoid with three orthogonal semi-axes of symmetry $a, b$, and $c$. In the particle reference system, $\hat{\alpha}$ is diagonal and its eigenvalues are given by [28] $\alpha_{i}=4 \pi a b c\left(\epsilon-\epsilon_{\mathrm{m}}\right) /\left(3 \epsilon_{\mathrm{m}}+3 L_{i}\left(\epsilon-\epsilon_{\mathrm{m}}\right)\right)$, where $\epsilon$ is the dielectric permittivity of the particle, and $L_{i}$, with $i=a, b, c$, are dimensionless quantities defined by the particle geometry (see Ref. [29], Sec. S1.i). For an arbitrary particle orientation in the laboratory system, the polarizability tensor can be transformed using $\hat{\alpha}=\hat{M}^{-1} \hat{\alpha}^{\prime} \hat{M}$, where $\hat{M}$ is a rotation matrix.

The interference of the reflected probe field with the reference field is calculated using $E_{2 r}^{ \pm}=\left(\mathbf{E}_{R}^{ \pm}\right)^{*} \cdot \hat{\alpha} \mathbf{E}_{2}^{+}$, where $\hat{\alpha} \mathbf{E}_{2}^{+}$is the particle-induced polarization for a $\sigma^{+}$ polarized incident probe field (we dropped the constant $\epsilon_{0} \epsilon_{\mathrm{m}}$ in $\mathbf{p}$ for brevity), and $\mathbf{E}_{R}^{ \pm}$are reference fields equal to left $(+)$ and right $(-)$ circularly polarized input fields. The technique is configured such that the optical modes of probe and reference fields are matched; hence, $\mathbf{E}_{R}$ was calculated as the field distribution in the focal region in the same way as $\mathbf{E}_{2}$ (see the Appendix) and back-propagated via time reversal. Similarly, the FWM interference is calculated as $E_{\mathrm{FWM}}^{ \pm}=\left(\mathbf{E}_{R}^{ \pm}\right)^{*} \cdot \delta \hat{\alpha} \mathbf{E}_{2}^{+}$. Here, $\delta \hat{\alpha}$ is the pump-induced change of the particle polarizability, which we have modeled as described in our previous work [27]. Briefly, $\delta \hat{\alpha}$ arises from the transient change of the electron and lattice temperature following the absorption of the pump pulse by the NP. Note that $\delta \hat{\alpha}$ depends on the pump fluence at the NP, on the particle absorption cross section, and on the delay time between pump and probe pulses (see also Ref. [29], Figs. S2 and S10). The simulations in Fig. 2 were performed to reproduce the experimentally measured FWM signal strength on a 30 -nm-radius gold NP at $\tau=0.5 \mathrm{ps}$ as shown later (note that $\tau \sim 0.5 \mathrm{ps}$ is the delay for which the FWM amplitude reaches its maximum as a result of the ultrafast heating of the electron gas [27]).

Here, $E_{2 r}^{ \pm}$and $E_{\mathrm{FWM}}^{ \pm}$have amplitudes and phases as a function of particle position in the focal region, as shown in Fig. 2 for the case of a perfectly spherical NP. Similar to the spatial distributions of the focused field shown in Fig. 1(b), $E_{2 r}^{-}$and $E_{\mathrm{FWM}}^{-}$form optical vortices of $l=2$ topological charge. The ratio $E_{2 r}^{-} / E_{2 r}^{+}$is also shown with its amplitude $A_{2 r}^{-} / A_{2 r}^{+}$and phase $\Phi_{2 r}^{-}-\Phi_{2 r}^{+}$. The FWM field distribution has a narrower PSF than the reflection, as expected from the third-order nonlinearity. The phase of $E_{\mathrm{FWM}}$ is shifted compared to $E_{2 r}$ because of the phase difference between $\delta \hat{\alpha}$ and $\hat{\alpha}$. Slices along the axial direction $(z)$ are shown for $E_{\mathrm{FWM}}$ (a similar behavior is observed for $E_{2 \mathrm{r}}$, see Ref. [29], Fig. S1). Of specific importance for the localization of the NP in $3 \mathrm{D}$ are the amplitude and phase of the FWM field ratio $E_{\mathrm{FWM}}^{-} / E_{\mathrm{FWM}}^{+}$ and the phase of the co-polarized $E_{\mathrm{FWM}}^{+}$as highlighted by the red panels and sketch in Fig. 2.

The co-polarized FWM amplitude is much stronger (about 90 fold) than the cross-polarized FWM and has a phase that is independent of the lateral position over the PSF width. Conversely, along the $z$ axis, this phase is linear in the displacement between particle and center of the focus and can be used to determine the particle $z$ coordinate. This can be easily understood as due to the optical path-length difference between the particle and the observation point. For a plane wave of wave vector $k=2 \pi n / \lambda$ with $n$ refractive index in the medium, the phase would be $2 \mathrm{kz}$, the factor of 2 accounting for the back and forth path in reflection geometry. We find a linear relationship between $\Phi_{\mathrm{FWM}}^{+}$and $z$ (see Ref. [29], Fig. S3), with a slope $\partial z / \partial \Phi=38.8 \mathrm{~nm} / \mathrm{rad}$, slightly larger than $\lambda /(4 \pi n)=$ $28.8 \mathrm{~nm} / \mathrm{rad}$. This is due to the propagation of a focused beam with high NA where a Gouy phase shift occurs, reducing the wave vector in the axial direction due to the wave-vector spread in the lateral direction.

For the in-plane radial coordinate $R$ of the NP position relative to the focus position (see sketch in Fig. 2), we find that the FWM amplitude ratio $A_{\mathrm{FWM}}^{-} / A_{\mathrm{FWM}}^{+}$scales quadratically with $R$ up to $R \sim 60 \mathrm{~nm}$, such that this coordinate can be calculated as $R=R_{0} \sqrt{A_{\mathrm{FWM}}^{-} / A_{\mathrm{FWM}}^{+}}$. Notably, by using the FWM ratio, the retrieved $R$ is independent of the pump, probe, and reference powers, and of the NP size (in the dipole approximation). Conversely, $R_{0}$ is specific to the NA of the microscope objective used and the probe beam fill factor (see Ref. [29], Fig. S4), and it decreases with increasing NA, showing that high NAs are required to localize the NP in $R$ down to small distances. Finally, the angular position coordinate $\varphi$ can be taken from the phase of the FWM ratio $\Theta=\Phi_{\mathrm{FWM}}^{-}-\Phi_{\mathrm{FWM}}^{+}$as $\varphi=\left(\Theta-\Theta_{0}\right) / 2$ (see also Ref. [29], Fig. S3).

In essence, using these relationships, we can locate the NP in 3D $(z, R$, and $\varphi)$ by scanless polarization-resolved and phase-resolved FWM acquisition at a single spatial point. Note that there is a $\pi$ ambiguity in the angular position coordinate $\varphi$ due to the scaling of the FWM ratio phase proportional to $2 \varphi$. This corresponds to an inversion of the position in the $(x, y)$ plane. In practical experiments, this ambiguity can be overcome by tracking the NP position over time, assuming a continuous motion, or by using an in situ calibration (see Ref. [29], Sec. S2 x.vi). 


\section{BACKGROUND-FREE FOUR-WAVE-MIXING DETECTION}

Prior to experimentally quantifying the nanometric localization precision, it is important to emphasize that our FWM detection is background-free even in scattering and/or autofluorescing environments, making it applicable to imaging single small NPs inside cells, surpassing other methods reported in the literature. Localizing single plasmonic NPs with nanometric precision even at submillisecond exposure times can be achieved in an optically clear environment with simpler techniques such as dark-field microscopy [7] and interferometric scattering microscopy [9]. However, since these techniques use the linear response of the NP, they are substantially affected by endogenous scattering and fluorescence, which severely limit their practical applicability in heterogeneous biological environments.

To exemplify this point, Fig. 3 (top panels) shows fixed HeLa cells that have internalized gold NPs of 20 -nm radius, imaged with FWM using a 1.45 NA oil-immersion objective. High-resolution DIC microscopy was available in the same instrument (for details see Ref. [29], Secs. S2.iii and S2.vii). Figure 3(a) shows the DIC image of a group of
HeLa cells on which reflection and FWM imaging was performed in the region highlighted by the dashed frame. The co-circularly polarized reflection image $A_{2 r}^{+}$shown in Fig. 3(b) correlates with the cell contour seen in DIC and shows a spatially varying contrast due to thickness and refractive index inhomogeneities in the sample. Even with a particle diameter as large as $40 \mathrm{~nm}$, gold NPs are not distinguished from the cellular contrast in DIC or in the $A_{2 \mathrm{r}}^{+}$ reflection image. Detecting the cross-polarized reflection $A_{2 r}^{-}$[Fig. 3(c)], which has been suggested as a way to improve contrast [30], is still severely affected by the cellular scattering background. On the contrary, the cocircularly polarized FWM amplitude $A_{\mathrm{FWM}}^{+}$shown in Fig. 3(d) as a maximum intensity projection over a $6-\mu \mathrm{m}$ $z$ stack is background-free (throughout the $z$ stack) and clearly indicates the location of single gold NPs in the cell. Notably, FWM acquisition can be performed simultaneously with confocal fluorescence microscopy for correlative co-localization analysis, as shown in Ref. [29], Fig. S11.

Figure 3 (bottom panel) shows a single 25-nm-radius gold NP embedded in a dense $(5 \% \mathrm{w} / \mathrm{v})$ agarose gel.

Single Au NPs in HeLa cells:
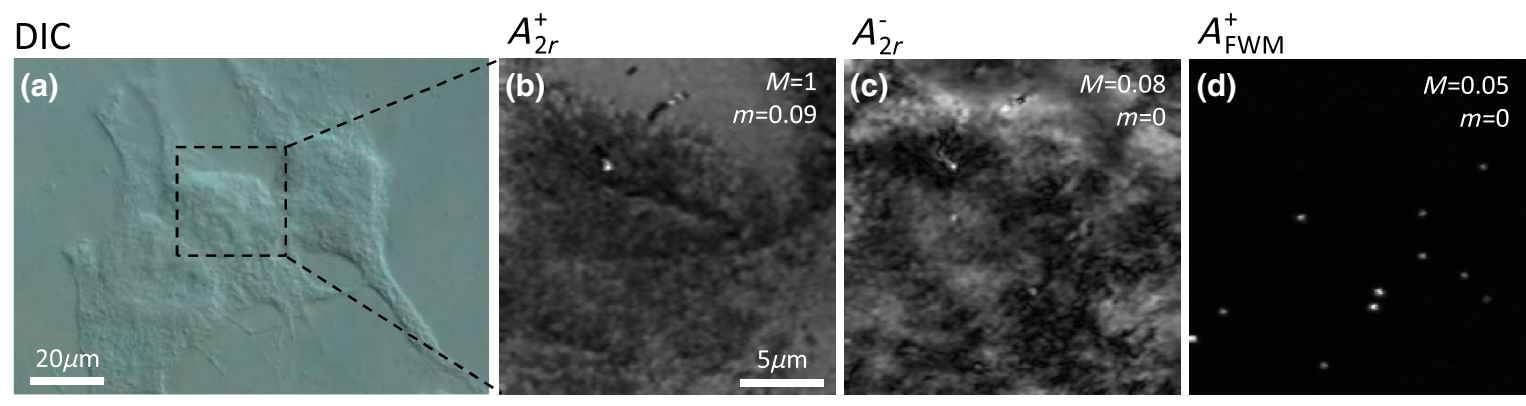

\section{Single Au NP in agar:}
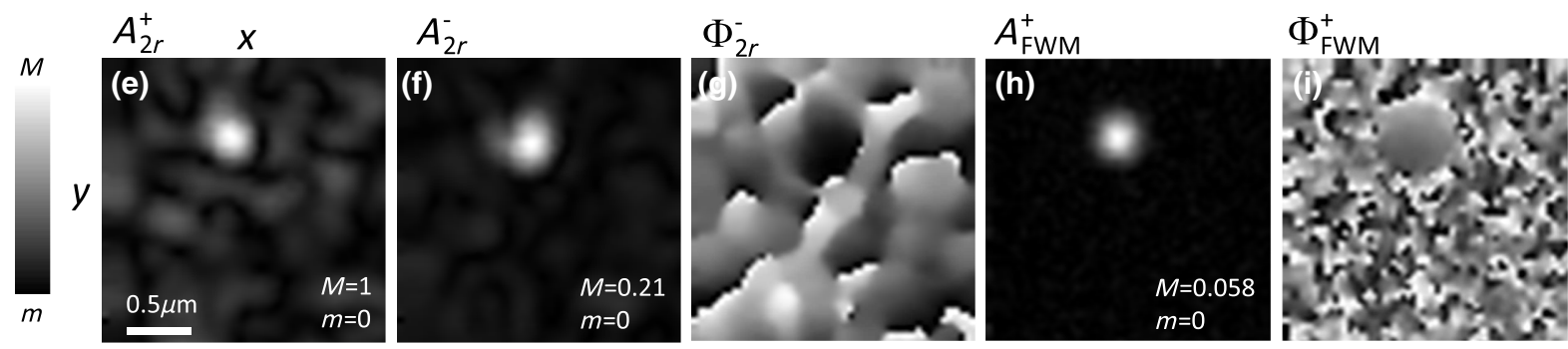

FIG. 3. Background-free FWM detection of single NPs in heterogeneous environments. Top panels: Fixed HeLa cells that have internalized gold NPs of $20 \mathrm{~nm}$ radius imaged by (a) differential interference contrast (DIC) microscopy, (b) co-circularly polarized reflection amplitude $A_{2 r}^{+}$, (c) cross-circularly polarized reflection amplitude $A_{2 r}^{-}$, and (d) co-circularly polarized FWM amplitude $A_{\mathrm{FWM}}^{+}$. FWM was acquired with a pump-probe delay time of $0.5 \mathrm{ps}$, pump (probe) power at the sample of $30 \mu \mathrm{W}(15 \mu \mathrm{W}), 2$-ms-pixel dwell time, pixel size in plane of $95 \mathrm{~nm}$ and $z$ stacks over $6 \mu \mathrm{m}$ in $250-\mathrm{nm} z$ steps. FWM is shown as a maximum intensity projection over the $z$ stack, while the reflection is on a single $(x, y)$ plane (scanning the sample position). Bottom panels: Single $(x, y)$-plane image of a 25-nm-radius gold NP in 5\% agarose gel, via (e) the co-circularly polarized reflection amplitude $A_{2 r}^{+}$, (f) cross-circularly polarized reflection amplitude $A_{2 r}^{-}$, and (g) phase $\Phi_{2 r}^{-}$, (h) co-circularly polarized FWM amplitude $A_{\mathrm{FWM}}^{+}$, and (i) phase $\Phi_{\mathrm{FWM}}^{+}$. FWM was acquired with 0.5 -ms-pixel dwell time and pixel size in plane of $38 \mathrm{~nm}$. Grey scales are linear from $-\pi$ to $\pi$ for all phases and from $m$ to $M$ for field amplitudes, as indicated. 
The scattering from the gel is visible as a structured background in the co-circularly polarized reflection image $A_{2 r}^{+}$in Fig. 3(e). Detecting the cross-polarized reflection [Fig. 3(f)] again does not eliminate the background. With the reflection amplitude scaling as the volume of the particle, a $15 \mathrm{~nm}$ radius NP would be indistinguishable from the background in the present case. Furthermore, the interference with the background limits the accuracy of particle localization. Notably, the phase $\Phi_{2 r}^{-}$is also severely affected by the scattering from the gel. Conversely, the FWM amplitude $A_{\mathrm{FWM}}^{+}$and phase $\Phi_{\mathrm{FWM}}^{+}$shown in Figs. 3(h) and 3(i) are background-free (as can be seen by the random phase outside the particle) and clearly resolve the NP despite the heterogeneous surroundings.

\section{EXPERIMENTAL LOCALIZATION: ROLE OF NANOPARTICLE ELLIPTICITY}

To experimentally quantify the nanometric localization and its precision due to photon shot noise, we start by examining a single, nominally spherical, 30 -nm-radius gold NP drop cast onto a glass coverslip and immersed in silicon oil, using an index-matched 1.45 NA oil-immersion objective.

The experimental data shown in Fig. 4 are scans of the $(x, y, z)$ sample position. They reveal all main features seen in the calculations, namely, a ringlike spatial distribution in plane of the amplitude ratio $A_{\mathrm{FWM}}^{-} / A_{\mathrm{FWM}}^{+}$and a phase of the cross-polarized component and, in turn, of the FWM ratio, rotating twice from 0 to $2 \pi$ along the in-plane polar angle. Similar results, with a wider PSF, as expected, are observed in the reflection components (see Ref. [29], Fig. S12). A main difference from the calculations is the observation of two displaced nodes, rather than a single central node, in the amplitude of the cross-polarized component, and in turn, a nearly constant phase of the cross-polarized component in the central area. The FWM ratio resolved across different axial planes is also shown in Fig. 4. We observe that, besides these minima, the axially resolved distributions agree with the calculated behavior of a linear relationship between $\Phi_{\mathrm{FWM}}^{+}$and $z$ and a $z$-dependent $\Theta_{0}$ manifesting as a rotated phase pattern of $\Theta=\Phi_{\mathrm{FWM}}^{-}-\Phi_{\mathrm{FWM}}^{+}$for different $z$ planes.

Notably, by measuring more than 50 particles in the sample, we could not find any pattern forming an $l=2$ optical vortex as simulated in Fig. 2. Different particles showed minima with different relative displacement, single displaced minima, or no minima within the spatial range of a sufficient signal-to-noise ratio (see Ref. [29], Fig. S13), suggesting that these minima are related to physical differences between particles rather than the effect of objective aberrations and/or misalignment of the setup, which we estimate to contribute to less than $1 \%$ of the amplitude ratio (see Ref. [29], Sec. S2.ix). Indeed, we can explain these experimental findings by assuming a small-particle asphericity. This is shown in the calculations in Fig. 4 where we used an ellipsoid nanoparticle with semi-axes $a=30.135 \mathrm{~nm}$ and $b=c=30 \mathrm{~nm}$ along the $x$, $y$, and $z$ axes in the particle reference system, which is rotated in plane relative to the laboratory system (see also Ref. [29], Figs. S5 and S6). It is remarkable that an asymmetry of only $0.5 \%$ in aspect ratio, or about one atomic layer of gold, manifests as a significant perturbation of the cross-polarized field patterns compared to the spherical case. With such sensitivity to asymmetry, the lack of experimentally observed patterns corresponding to a perfectly spherical particle is not surprising, considering the real particle morphology as observed in transmission electron microscopy (TEM) for this sample (see top inset in Fig. 4 and Ref. [29], Fig. S8).

The shot noise in the experiment, as well as the deviation from perfect sphericity, affects the localization precision. This is analyzed in Fig. 5. First, we performed simulations as in Fig. 2 for a perfectly spherical particle including experimental shot noise. The experimental shot noise was evaluated by taking the statistical distribution of the measured FWM field (in both the in-phase Re and inquadrature Im components) in a spatial region away from the particle, where no FWM is detectable. The standard deviation $\sigma$ of this distribution was deduced and was found to be identical in both components, as well as for the copolarized and cross-polarized components, as expected for an experimental noise dominated by the shot noise in the reference beam (see Ref. [29], Fig. S9, for the dependence of $\sigma$ on the power in the reference beam). A relative noise figure was defined as $\sigma / A_{0}$, with $A_{0}$ being the maximum measured value of the co-polarized FWM field amplitude. The simulations were performed using a statistical distribution of the FWM field values (Re and Im components) at each spatial pixel having the same relative noise $\sigma / A_{0}$ as the experimental data.

To quantify the uncertainty in the localization of the particle coordinates, we then calculated the deviation $\Delta=$ $\sqrt{\left(x-x_{s}\right)^{2}+\left(y-y_{s}\right)^{2}+\left(z-z_{s}\right)^{2}}$ between the set position of the NP in 3D $\left(x_{s}, y_{s}, z_{s}\right)$ and the deduced position $(x, y, z)$ using the FWM field amplitude and phase as detailed above. The resulting $\Delta$ in the focal plane $(x, y, 0)$ and in the $(x, 0, z)$ cross section along the axial direction are shown in Fig. 5(a) for the ideal case as in Fig. 2, and in Fig. 5(b) when adding a relative noise of $0.07 \%$ corresponding to the experiment in Fig. 4. In the ideal case, $\Delta \neq 0$ is a measure of the validity of the assumed dependencies, in particular, the quadratic behavior in the radial coordinate $R$, which becomes inaccurate for $R>$ $60 \mathrm{~nm}$ as discussed. This deviation can be easily removed by including an $R^{4}$ term (see Ref. [29], Fig. S3). Notably, in the region where the assumed trends are valid, we observe that adding the experimental noise results in a localization uncertainty of less than $20 \mathrm{~nm}$. Increasing the relative noise by 1 order of magnitude to $0.7 \%$ results in a localization uncertainty of about $50 \mathrm{~nm}$, as shown in Fig. 5(c). We emphasize that $\Delta$ is dominated by the in-plane 
Experiment: Single Au NP on glass surface
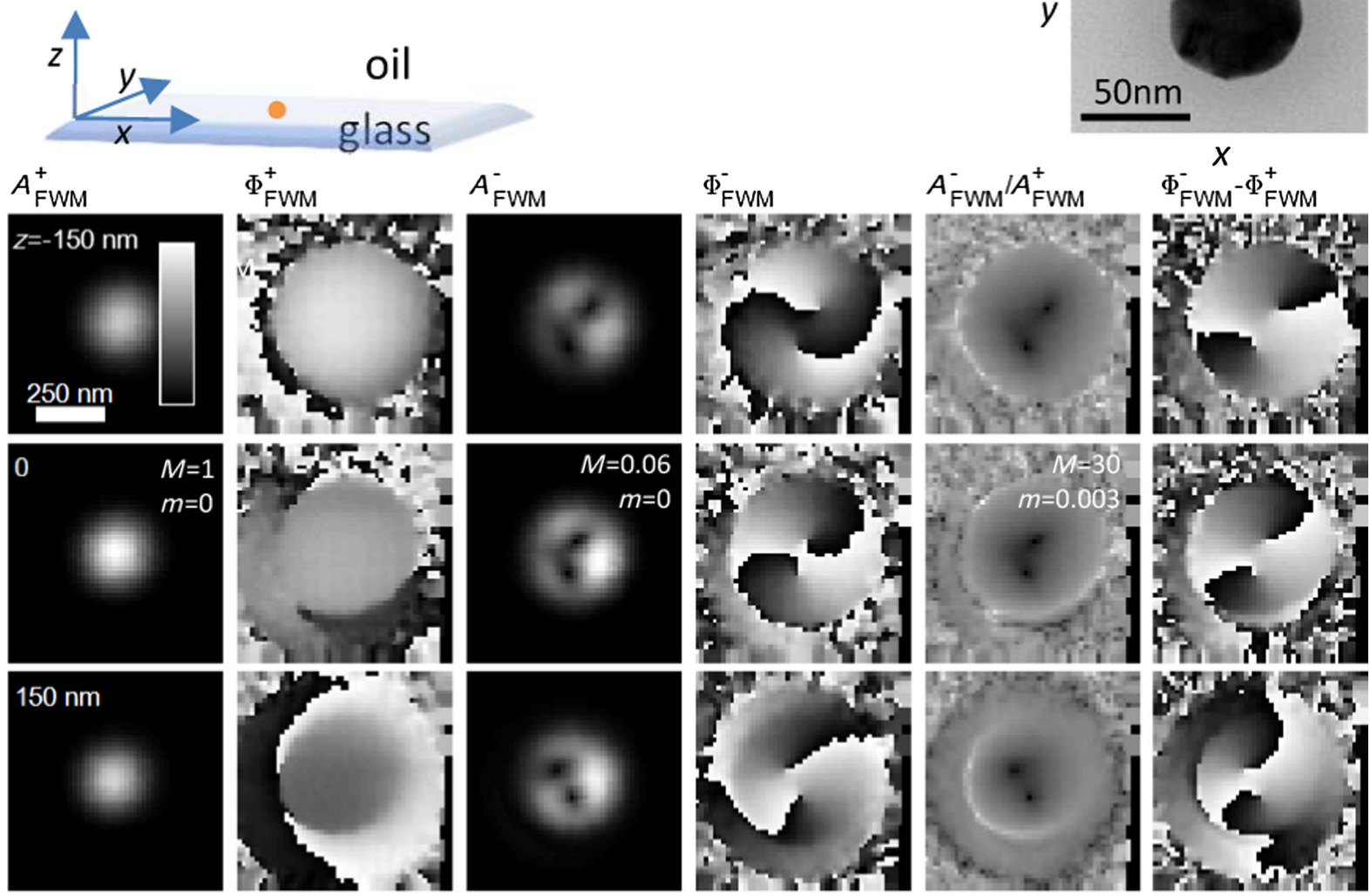

\section{Calculations: Elliptical Au NP}
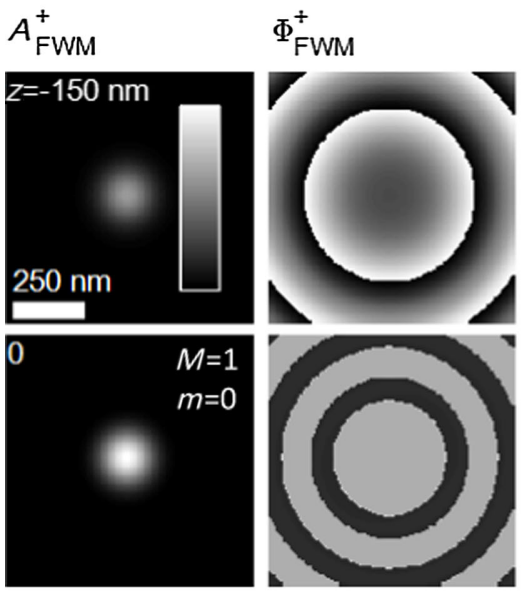

$A_{\text {FWM }}^{-}$
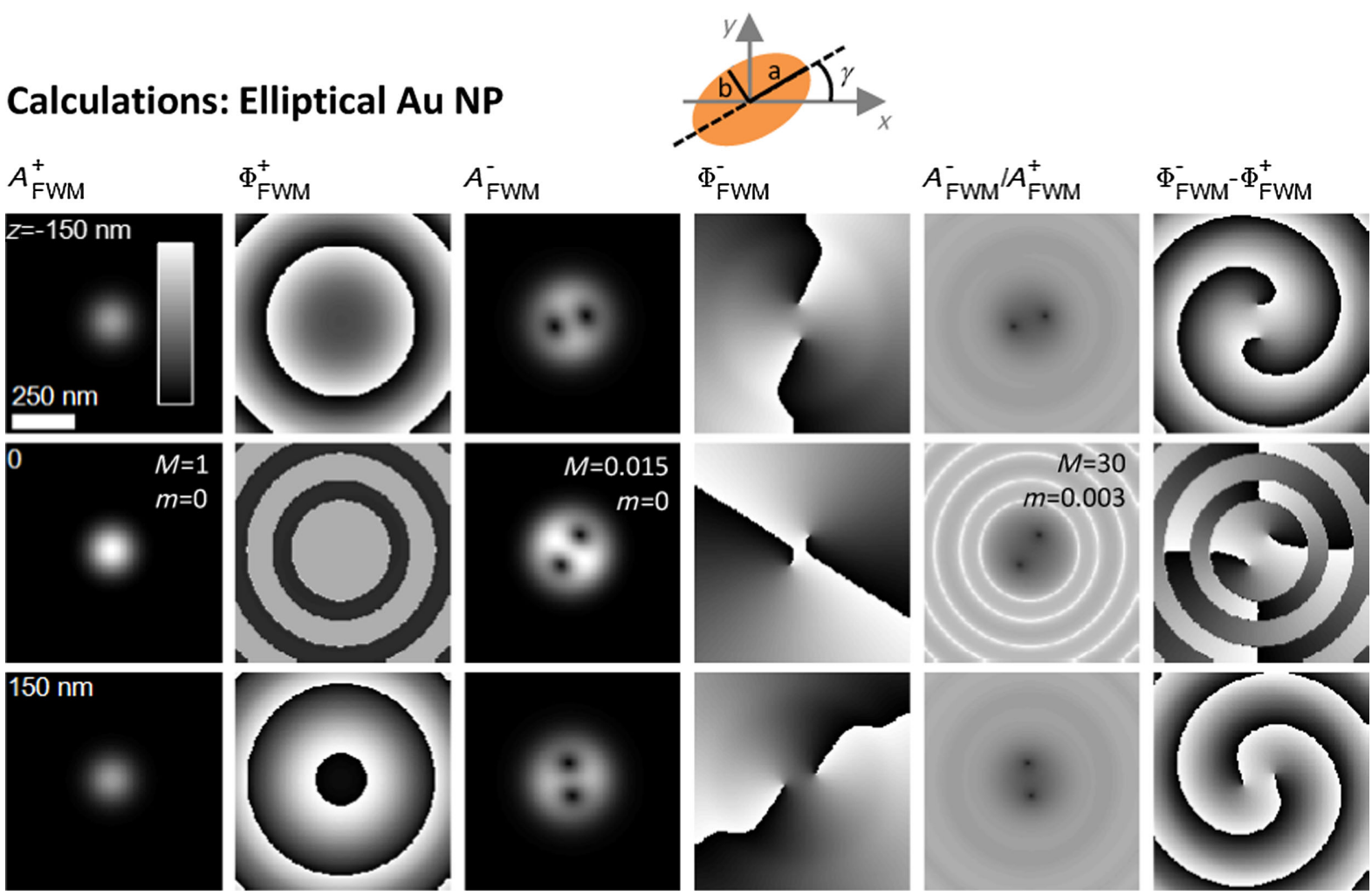

FIG. 4. FWM of a single NP attached on glass. We show the amplitude and phase as a function of NP position in the $(x, y)$ plane at different axial positions $z$. The linear grey scale is from $-\pi$ to $\pi$ for phases and from $m$ to $M$ for field amplitudes. The amplitude ratio is on a logarithmic scale over 4 orders of magnitude. Experiment: The top inset shows a sketch of the sample and a TEM image of a typical NP from the batch used. The pump (probe) power at the sample was $18 \mu \mathrm{W}(9 \mu \mathrm{W})$, with a 3-ms-pixel dwell time and 0.5-ps pump-probe delay time; the pixel size in plane (axial) was $17 \mathrm{~nm}(75 \mathrm{~nm})$. Calculations assume particle asphericity in plane with semi-axes $a=30.135 \mathrm{~nm}$ and $b=30 \mathrm{~nm}$ (see text). 
(a)

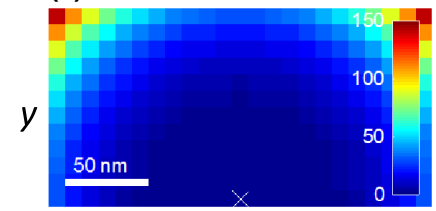

(b)

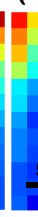

(c)

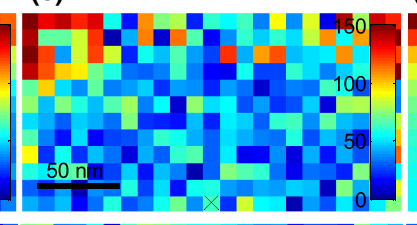

(d)

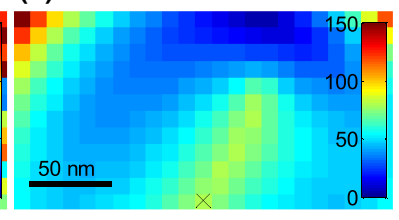

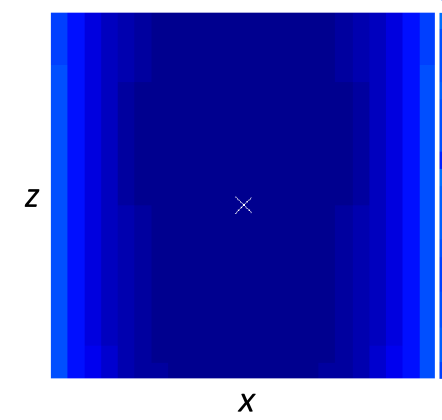
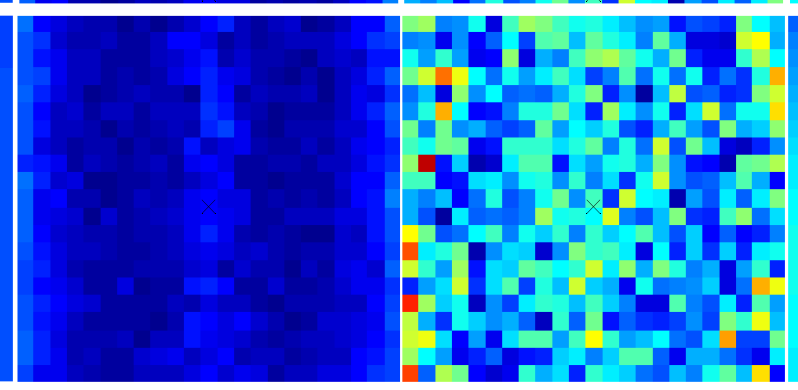

FIG. 5. Position localization precision. Difference between the set position of the nanoparticle in 3D and the deduced position using the FWM field amplitude and phase with the coordinate reconstruction parameters as described in the text. The color bar gives the values of this difference in nanometers. The top panels show maps in the $(x, y)$ plane, and the bottom panels are axial $(x, z)$ maps. The cross shows the position of the focus center. (a) Spherical particle without shot noise. (b) Spherical particle with relative shot noise of $0.07 \%$ as in the experiment in Fig. 4. (c) Spherical particle with relative shot noise of $0.7 \%$. (d) Asymmetric particle with semi-axis $a=30.135 \mathrm{~nm}$ and $b=c=30 \mathrm{~nm}$, as in the simulations in Fig. 4.

uncertainty, i.e., $\Delta=\sqrt{\left(x-x_{\mathrm{s}}\right)^{2}+\left(y-y_{\mathrm{s}}\right)^{2}+\left(z-z_{\mathrm{s}}\right)^{2}} \cong$ $\sqrt{\left(x-x_{\mathrm{s}}\right)^{2}+\left(y-y_{\mathrm{s}}\right)^{2}}$. In fact, considering that the axial direction $z$ is determined directly by the slope of $\Phi_{\text {FWM }}^{+}$and does not involve the cross-polarized FWM, we find $z$ localization accuracies as small as $0.3 \mathrm{~nm}$ for $0.7 \%$ noise in $\Phi_{\text {FWM }}^{+}$.

Note that the relative shot noise in the experiments scales as $1 /\left(V \sqrt{t} I_{1} \sqrt{I_{2}}\right)$, where $V$ is the particle volume (in the Rayleigh regime), $I_{1}\left(I_{2}\right)$ is the intensity of the pump (probe) beam at the sample, and $t$ is the acquisition time. It can therefore be adjusted according to the requirements. For example, a 15-nm-radius NP imaged under the experimental conditions as in Fig. 4 would give rise to an eightfold increase in relative noise, hence still maintaining the localization uncertainty to below $50 \mathrm{~nm}$. These results elucidate that a localization precision of below $20 \mathrm{~nm}$ in plane and below $1 \mathrm{~nm}$ in the axial direction is achievable with the proposed method with a realistic shot-noise level as in the experiment.

However, the lack of particle symmetry is a limitation for the in-plane localization. Introducing the particle asymmetry, without shot noise, as calculated in Fig. 4, results in a significant deviation of $\Delta \sim 100 \mathrm{~nm}$ in the central area due to the lack of a central node in the cross-polarized FWM amplitude [see Fig. 5(d)]. On the other hand, it is remarkable how sensitive the described FWM technique is to particle asymmetry, which can be used as a new tool to detect particle ellipticity down to $a / b-1=10^{-4}$ (corresponding to atomic precision comparable to TEM) as well as particle orientation. We find that the FWM amplitude ratio in the focus center scales linearly with the particle ellipticity and that the phase of the FWM ratio in the focus center scales with the in-plane particle orientation angle (which for the data in Fig. 4 was found to be $\gamma=150^{\circ}$; see Ref. [29], Figs. S5 and S6).

The limitation of particle asymmetry can be overcome by improving colloidal fabrication techniques. For example, it has been reported in the literature that gold nanorods can be synthesized as single crystals without stacking faults or dislocations [31], and they can be shaped to become spherical particles under high-power femtosecond laser irradiation [32]. We also note that when examining gold NPs of 5-nm radius (see Ref. [29], Figs. S8 and S14), we found that a large proportion (about 70\%) have a FWM amplitude ratio $A_{\mathrm{FWM}}^{-} / A_{\mathrm{FWM}}^{+} \leq 0.02$ in the center of the focal plane, in this case limited by the signal-to-noise ratio rather than the particle asymmetry, suggesting that smaller NPs might be intrinsically more monocrystalline and hence symmetric.

Importantly, when NPs are not immobilized onto a surface but are freely rotating (a more relevant scenario for particle-tracking applications), we can expect that the acquired field components time averaged over a sufficiently long acquisition time would no longer be affected by the particle asymmetry since this is averaged upon rotation (the rotational diffusion constant in water at room temperature of a 30-nm-radius nanoparticle is about $10^{4} \mathrm{rad}^{2} / \mathrm{s}$ ) and would lead to a localization precision only limited by shot noise. This case is discussed in the next section.

\section{ROTATIONAL AVERAGING}

To mimic a relevant biological environment such as the cytosolic network, as well as having single NPs freely rotating but not diffusing out of focus, gold NPs of 25-nm 
radius were embedded in a dense (5\% w/v) agarose gel in water (see Ref. [29], Sec. S2.ii) and measured using an index-matched 1.27 NA water-immersion objective. For these experiments, the focused size of the pump beam was increased by a factor of 2 (by under filling the back focal aperture), to enlarge the region where a single NP could diffuse while still being excited by the pump field, hence giving rise to FWM (this also increases the maximum crosspolarized FWM amplitude). Conversely, the probe beam was tightly focused to exploit the full NA of the objective and in turn exhibit an $l=2$ optical vortex in the crosscircularly polarized component as calculated in Fig. 2.

Figure 6(a) shows an $x y$ scan of the measured cross and co-circularly polarized FWM field in the focal plane, and the corresponding ratio in amplitude and phase, on a single NP while freely rotating but being enclosed in a tight pocket of the agar network, resulting in a negligible average translation during the measurement time (for an estimate of the size of the pocket, see Ref. [29], Sec. S2.xiv). As expected, the rotational averaging enables the observation of an optical vortex with a central amplitude node in the cross-circularly polarized FWM. Surprisingly, however, the phase pattern reveals an $l=1$ vortex, as opposed to the predicted $l=2$; i.e., the phase directly changes with the in-plane polar angle instead of with twice the angle. Similar results are observed in the reflected field components, albeit with poorer contrast compared to FWM due to the reflection background from the gel (see Ref. [29],

(a) Experiment: Single Au NP rotating in agar

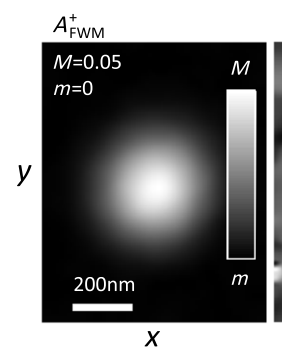

(b) Experimental position tracking in 3D over time:
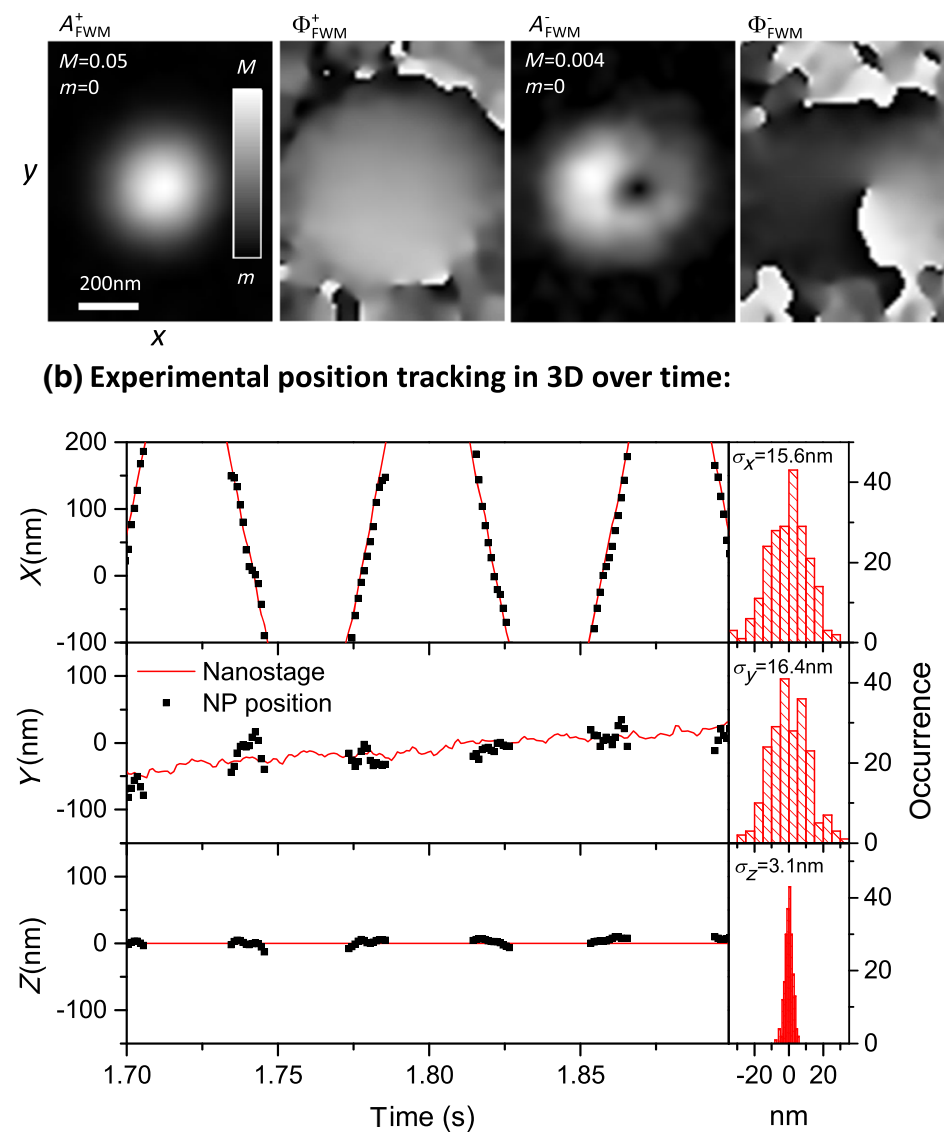

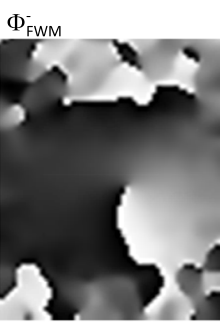

\section{(c) Calculations}

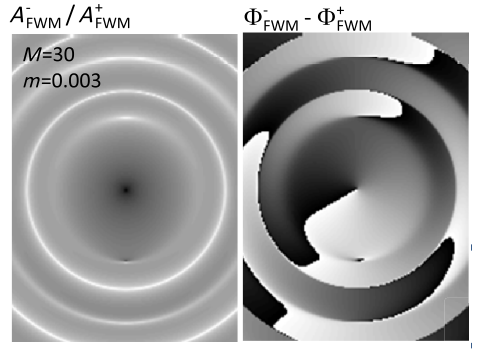

(d) Sketch of NP encaged in agar

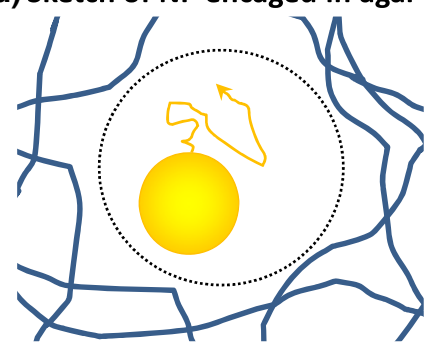

FIG. 6. Rotational averaging of nanoparticle asymmetry. We show single 25-nm-radius gold NP freely rotating while encaged in an agarose gel pocket (see sketch). (a) Experimental $x y$ scan of the cross- and co-circularly polarized FWM field in the focal plane, using a 1.27 NA water-immersion objective. We show the linear grey scale from $-\pi$ to $\pi$ for phases and from $m$ to $M$ for field amplitudes, with $M$ given relative to the maximum $A_{2 r}^{+}$. The amplitude ratio is on a logarithmic scale. The pump (probe) power at the sample was $70 \mu \mathrm{W}$ $(10 \mu \mathrm{W})$ with pump (probe) filling factor 2.15 (0.97). Measurements were performed with 0.5-ms-pixel dwell time, 0.5-ps pump-probe delay time, and 13-nm pixel size in plane. Data are shown as spatial averages over an effective area of $3 \times 3$ pixels. (b) Time traces of the retrieved-particle-position coordinates in 3D from the measured FWM amplitude and phase (symbols) compared with the coordinates from the scanning piezoelectric sample stage (lines). Traces are binned to an equivalent $1 \mathrm{~ms}$ acquisition time per point. After subtracting the stage coordinates, histograms of the difference between consecutive points are shown, and the corresponding localization precisions are indicated (see text). (c) Calculated FWM field ratio assuming a polarization tensor that projects the longitudinally polarized field component into the $(x, y)$ plane (see text). 
Fig. S15). Calculations show that for an incident circularly polarized field, the longitudinally polarized component $\left(E_{z}\right)$ in the focus of a high NA objective has this symmetry, and we can reproduce the experimental findings by introducing a particle polarizability tensor that projects the longitudinal component into the $x y$ plane (see Ref. [29], Sec. S1.vii). Conversely, it is not possible to reproduce the experimental findings by assuming a nonrotating, randomly oriented, asymmetric particle (see Ref. [29], Sec. S2.xv); hence, free rotation is key to the pattern observed in Fig. 6(a). Since the only particle asymmetry that does not average upon rotation is $3 \mathrm{D}$ chirality, we suggest that this response is a manifestation of chirality (albeit a detailed theoretical understanding of the nonlinear optical response of a chiral particle is beyond the scope of this work). We note that these quasispherical NPs have irregularities of a few atom clusters, which can lead to symmetry breaking, as already seen for their ellipticity, and thus also 3D chirality (see TEM in Ref. [29], Fig. S8). Notably, an $l=1$ optical vortex offers a simpler dependence for the retrieval of the NP position coordinates, with a FWM amplitude ratio $\left(A_{\mathrm{FWM}}^{-} / A_{\mathrm{FWM}}^{+}\right)=R / R_{0}$, i.e., scaling linearly with $R$, and a phase $\Theta=\Phi_{\mathrm{FWM}}^{-}-\Phi_{\mathrm{FWM}}^{+}$directly changing with the polar angle $\varphi$ (see Ref. [29], Fig. S16). Using these dependencies (alongside the axial position $z$ given by $\Phi_{\mathrm{FWM}}^{+}$as previously shown), we have retrieved the NP position coordinates in 3D for the scan shown in Fig. 6(a) and compared them with the position coordinates recorded from the scanning piezoelectric sample stage (for $x$ and $y$, while $z$ was not scanned, and for which the nominal position is indicated). This result is shown in Fig. 6(b). To quantify the localization precision, we then evaluated the difference $\left(d_{i}\right)$ between the retrieved NP coordinate and the stage position for each point $(i)$ in the trace, and we calculated the standard deviation of the corresponding histograms (see Ref. [29], Fig. S17), which resulted in $23 \mathrm{~nm}, 21 \mathrm{~nm}$, and $14 \mathrm{~nm}$ for $x, y$, and $z$, respectively. These values are a combination of precision and accuracy, as they include the effects of systematic drifts in addition to shot noise. To remove the contribution of drifts, we then followed a procedure similar to the "stepsize statistics" used in Ref. [33]; namely, we calculated the difference $D_{i}=d_{i+1}-d_{i}$ between consecutive points, and the corresponding histograms are shown in Fig. 6(b). By evaluating the difference, the standard deviation of these histograms is a factor $\sqrt{2}$ larger than for individual measurements and additionally must be corrected for the degree of correlation in the measurements of consecutive points (see Ref. [29], Fig. S17). The resulting measured precisions are $16 \mathrm{~nm}$ in $x, y$ and $3 \mathrm{~nm}$ in $z$, at $1 \mathrm{~ms}$ acquisition time per point. The values in plane are consistent with the estimated shot-noise limit, which, owing to the simpler linear dependencies for the retrieval of the NP position coordinates in this case, can be calculated as $R_{0} * \sigma / A_{\mathrm{FWM}}^{+}$, with $R_{0}=1.33 \mu \mathrm{m}$ being the coordinate retrieval parameter in plane and the relative shot noise $\sigma / A_{\mathrm{FWM}}^{+}$varying between $0.7 \%$ and $1.4 \%$ (from the variation of $A_{\mathrm{FWM}}^{+}$in the trace). Conversely, the measured precision in $z$ is larger than the estimated shot-noise limit using $37 *\left(\sigma / A_{\mathrm{FWM}}^{+}\right) \mathrm{nm}$ (from $\partial z / \partial \Phi=37 \mathrm{~nm} / \mathrm{rad}$ with the water-immersion objective; see Fig. S16), which ranges from $0.3 \mathrm{~nm}$ to $0.5 \mathrm{~nm}$. By analyzing the precision of the nanopositioning stage, we found a 3.2-nm standard deviation (see Fig. S17), which is comparable to the precision measured in $z$ and therefore attributed to the stage motion.

\section{SINGLE-PARTICLE TRACKING}

A demonstration of the practical applicability of the method for scanless single-particle tracking in 3D is shown in Fig. 7 on gold NPs of 25-nm radius embedded in a dense $(5 \% \mathrm{w} / \mathrm{v})$ agarose gel in water, which provides a heterogeneous environment as shown in Fig. 3 (bottom panel). For practical purposes, we implemented a simple in situ calibration of the in-plane NP position coordinates without the need for prior knowledge and/or characterization of the particle optical response. As shown in Fig. 7(a), we apply a small oscillation of known amplitude $(16 \mathrm{~nm})$ and frequency $(25 \mathrm{~Hz})$ to one axis of the sample stage. When the FWM field ratio from the NP encodes the NP in-plane position as discussed in the previous section, this oscillation is detected in the measured $E_{\mathrm{FWM}}^{-} / E_{\mathrm{FWM}}^{+}$with an amplitude and a phase that we can use to directly calibrate the FWM field ratio in terms of in-plane position coordinates of known size and direction (see also Ref. [29], Sec. S2 x.vi). Figure 7(a) shows the power spectrum of the Fourier transform of $E_{\mathrm{FWM}}^{-} / E_{\mathrm{FWM}}^{+}$, which exhibits a peak at this modulation frequency. The correspondingly calibrated particle-position coordinates over time are shown in Fig. 7(b). Conversely, if for a NP we do not observe this oscillation in $E_{\mathrm{FWM}}^{-} / E_{\mathrm{FWM}}^{+}$, the FWM field ratio is a measure of the NP in-plane asymmetry and orientation. This case is shown in Figs. 7(c) and 7(d) (for the power spectrum, see Ref. [29], Sec. S2 x.vi). Importantly, we can still accurately measure the axial-position coordinate, which is directly given by $\Phi_{\mathrm{FWM}}^{+}$and does not involve the cross-polarized FWM, while now $E_{\mathrm{FWM}}^{-} / E_{\mathrm{FWM}}^{+}$encodes information on the NP asymmetry and orientation, rather than its in-plane position. Figure 7(c) shows examples of traces of the NP $z$ position acquired over several tens of seconds (with 2-ms point acquisition), from which we can see a "jumping behavior" around two preferred axial locations, suggesting the presence of two pockets in the agar gel separated by about $100 \mathrm{~nm}$. Figure 7(d) shows the $z$ position together with $E_{\mathrm{FWM}}^{-} / E_{\mathrm{FWM}}^{+}$(as real and imaginary parts) as a zoom over a time window [from the top trace in panel (c)] during which the NP got stuck in a corner, below the center of the lower pocket. From the strong and slowly varying FWM field ratio, the 
In situ calibration:

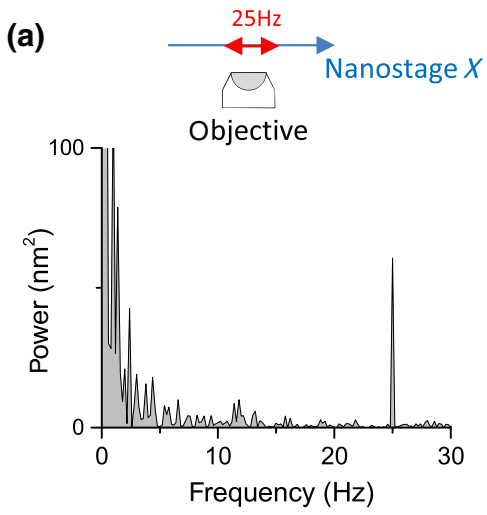

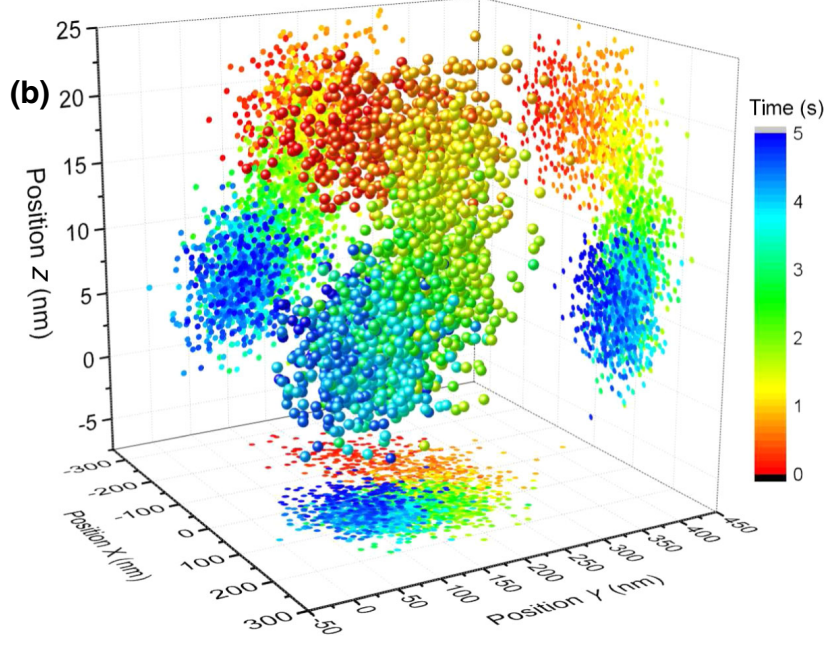

Confined particle diffusion between agar pockets :
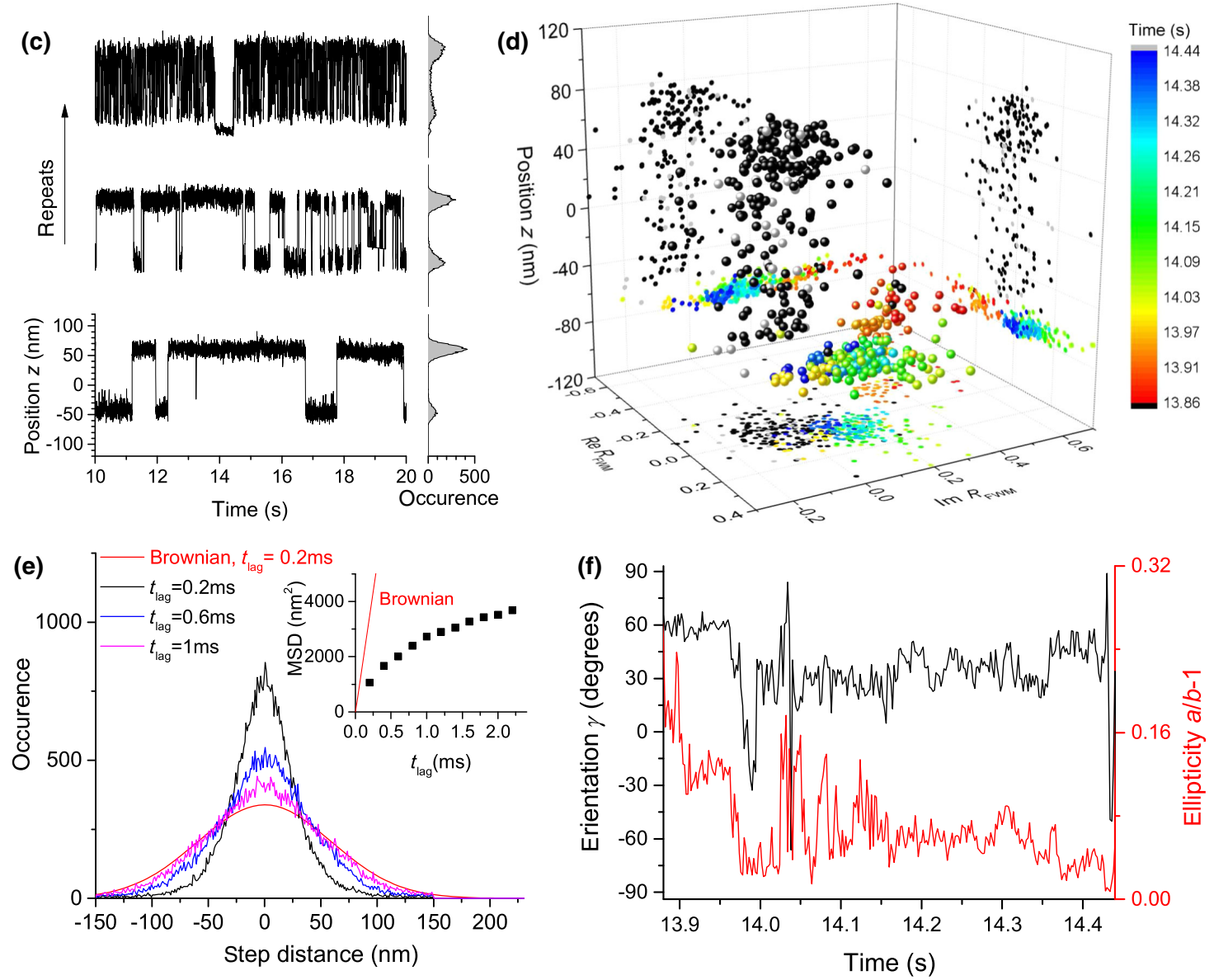

FIG. 7. In situ calibration and nanoparticle tracking. (a) Power spectrum of the FWM field ratio showing the $25 \mathrm{~Hz}$ oscillation imposed on the sample stage for calibration, as sketched. (b) Retrieved position coordinates versus time of a single $25 \mathrm{~nm}$ radius NP in agar (2 ms per point). (c) Axial-position time traces of a single gold NP while jumping between two gel pockets. (d) Zoom of top trace in panel (c), showing the axial position and FWM field ratio dominated by the NP asymmetry; the corresponding in-plane ellipticity and orientation are shown in panel (f). (e) Analysis of axial-position time traces acquired at $0.2 \mathrm{~ms}$ per point (see text).

corresponding time evolution of the NP in-plane orientation angle and aspect ratio is given in Fig. 7(f). Finally, Fig. 7(e) shows the analysis from an axial-position time trace acquired at high speed $(0.2 \mathrm{~ms}$ time per point, for over
$10 \mathrm{~s}$ ), indicating constrained diffusion. The traces were analyzed by calculating the mean square displacement (MSD) as a function of time lag $\left(t_{\text {lag }}\right)$ [1]. Histograms of the displacement for different $t_{\text {lag }}$ are shown in Fig. 7(e). 
The variance of the histogram gives the MSD, which is plotted versus $t_{\text {lag }}$ in the inset. For Brownian diffusion $\mathrm{MSD}=2 D t_{\mathrm{lag}}$ (for each dimension in space), with the free diffusion coefficient $D$ in water given in Ref. [29], Sec. S2.xiv. The resulting linear behavior is shown as a red line (and red Gaussian histogram) in Fig. 7(e). The observed sublinear dependence and saturation of MSD versus $t_{\text {lag }}$ indicates confined diffusion.

\section{DISCUSSION AND CONCLUSION}

We have shown a new method to determine the position of a single nonfluorescing gold NP with nanometric precision in three dimensions from scanless far-field optical measurements. The method is based on the interferometric detection of the polarization-resolved resonant FWM field in amplitude and phase, with a high numerical aperture objective. The displacement of the nanoparticle from the center focus in the axial direction is directly determined from the epidetected FWM field phase, while the in-plane displacement manifests as a cross-circularly polarized component because of the optical vortex field pattern in the focus of a high numerical aperture objective, the amplitude and phase of which enables accurate position retrieval.

Shape asymmetry of the NP of as little as $0.5 \%$ ellipticity, corresponding to about one atomic layer of gold, also induces a cross-circularly polarized field. This exceptional sensitivity to asymmetry eventually limits accurate position retrieval in plane. This can be overcome by observing a NP freely rotating, such that rotational averaging of the asymmetry occurs over the acquisition time.

Experimentally, we show that the FWM detection is completely background-free in scattering environments such as biological cells and that it outperforms existing methods such as reflectometry, scattering, and differential interference contrast. A localization uncertainty of 23-nm standard deviation in plane, and $14 \mathrm{~nm}$ axially, limited by systematic drifts, is shown with a single NP of $25-\mathrm{nm}$ radius freely rotating in an agarose gel, at an acquisition time of only $1 \mathrm{~ms}$. By analyzing the statistics of the difference between adjacent points, thus overcoming drifts, the precision is measured to be $16 \mathrm{~nm}$ in plane, consistent with the estimated shot noise. Axially, it is found to be $3 \mathrm{~nm}$, limited by the precision of the nanopositioning sample stage, and with an estimated shot-noise limit of $0.5 \mathrm{~nm}$. Smaller NPs can be measured by correspondingly increasing the intensity of the incident beams and/or the acquisition time. Notably, we find that, during free rotation, the cross-circularly polarized FWM field distribution in the focal plane is an $l=1$ optical vortex, as opposed to the predicted $l=2$ for a perfectly spherical particle, and we suggest that this is a manifestation of NP chirality not averaged during rotation.
To demonstrate the practical applicability of the method for single-particle tracking, we provide two examples of NPs diffusing in a dense agarose gel network. In one case, we show particle-position coordinates retrieved using an in situ calibration procedure and the corresponding tracking in 3D. In the second case, we show that when the crosscircularly polarized component is dominated by the effect of shape asymmetry, it can be used for tracking the in-plane asymmetry and orientation, while the axial-position coordinate provides information on the particle diffusion.

Ultimately, this method paves the way towards a new form of single-particle tracking, where not only the NP position but also its asymmetry, orientation, and chirality are detected with submillisecond time resolution, revealing much more information about the NP and its complex dynamics (e.g., hindered rotation) while moving and interacting within a disordered 3D environment.

Data availability Information about the data created during this research, including how to access it, is available from Cardiff University data archive at [38].

\section{ACKNOWLEDGMENTS}

This work was funded by the UK EPSRC Research Council (Grants No. EP/I005072/1, No. EP/I016260/1, No. EP/L001470/1, No. EP/J021334/1, and No. EP/ M028313/1) and by the EU (FP7 Grant No. ITNFINON 607842). P. B. acknowledges the Royal Society for funding. We acknowledge Lukas Payne for help in sample preparation and TEM characterization, and Peter Watson, Paul Moody, and Arwyn Jones for help in cell culture protocols.

P. B. and W. L. conceived the technique and designed the experiments. W. L. wrote the acquisition software. F. M. performed preliminary experiments and calculations. G. Z. performed the final calculations and the experiments on NPs attached onto glass. N. G. performed the experiments on cells and prepared NPs in agar. W. L. and P. B. performed the experiments on NPs in agar. P. B. wrote the manuscript. All authors discussed and interpreted the results and commented on the manuscript.

\section{APPENDIX: METHODS}

\section{FWM experimental setup}

Optical pulses of 150-fs duration centered at 550-nm wavelength with $\nu_{L}=80 \mathrm{MHz}$ repetition rate were provided by the signal output of an optical parametric oscillator (Newport/Spectra Physics, OPO Inspire HF 100) pumped by a frequency-doubled femtosecond Ti:Sa laser (Newport/Spectra Physics, Mai Tai HP). In the experiment, the amplitude modulation frequency of the pump beam was $\nu_{m}=0.4 \mathrm{MHz}$. The MO was either a $100 \times$ magnification oil-immersion objective of 1.45 NA (Nikon CFI Plan Apochromat lambda series) or a $60 \times$ magnification water-immersion objective of 1.27 NA 
(Nikon CFI Plan Apochromat lambda series, super resolution) mounted into a commercial inverted microscope stand (Nikon Ti-U). The sample was positioned with respect to the focal volume of the objective by an $x y z$ piezoelectric stage with nanometric position precision (MadCityLabs NanoLP200). A prism compressor was used to precompensate the chirp introduced by all the optics in the beam path, to achieve Fourier-limited pulses of 150-fs duration at the sample. Furthermore, since the reference beam does not travel through the microscope objective, glass blocks of known group-velocity dispersion were added to the reference beam path, in order to match the chirp introduced by the microscope optics and thus maximize the interference between the reflected FWM field and the reference field at the detector. $\mathrm{AOM}_{2}$ was driven at $\nu_{2}=82 \mathrm{MHz}$. $\mathrm{BS}_{1}$ is a $80: 20$ transmission: reflection splitter, transmitting most of the signal. We used balanced silicon photodiodes (Hamamatsu S5973-02) with home-built electronics and a high-frequency digital lock-in amplifier (Zürich Instruments HF2LI) providing six dual-phase demodulators, enabling us to detect, for both polarizations, the carrier at $\nu_{2}-\nu_{L}=2 \mathrm{MHz}$ and both sidebands at $\nu_{2} \pm \nu_{m}-\nu_{L}=2 \pm 0.4 \mathrm{MHz}$. This scheme overcomes the limitation in our previous work of using two separated lock-ins with associated relative phase offset [27], and it provides an intrinsic phase referencing and a noise reduction via the detection of both sidebands (see Ref. [29], Sec. S2.iv).

\section{Numerical simulations}

The field in the focal area is calculated using PSF Lab-a software package that allows calculation of the pointspread function of an aplanatic optical system for light that passes through stratified media [34]. Note that, as an alternative to numerical calculations, simplified formulas of the Debye-Wolf diffraction integral by means of a series of analytic functions that are physically meaningful can be found in Refs. [35-37], with the extended Nijboer-Zernike theory [35] being specifically suited to account for aberrations, and the multipole expansion in terms of vectorial spherical harmonics [36] providing a convenient decomposition to calculate light scattering by particles using the generalized Lorentz-Mie theory or the T-matrix approach. For the calculations in Figs. 2 and 4, the simulation parameters-wavelength $\lambda$, objective lens NA, coverslip thickness $d$, medium refractive index $n$, back-objective filling factor $\beta$-were chosen to match the actual experimental conditions in Fig. 4, namely, $\lambda=550 \mathrm{~nm}$, $\mathrm{NA}=1.45, d=0.17 \mathrm{~mm}, n=1.5185$, and $\beta=0.83$. The filling factor is defined as $\beta=a / w$, where $a$ is the aperture radius of the objective lens and $w$ is the Gaussian parameter in the electric-field radial dependence at the objective aperture $E=E_{0} e^{\left(-r^{2} / w^{2}\right)}$. For the calculations in Fig. 6, the simulation parameters were $\mathrm{NA}=1.27, n=1.333$, $\beta=2.15$ for the pump and $\beta=0.97$ for the probe beam.
The calculation was performed for a linear polarization of the incident field along the $x$ axis. This procedure results in a vectorial field at the focus called $\mathbf{E}^{x}(\mathbf{r})$. For the orthogonal linear incident polarization, the results were rotated counterclockwise to obtain $\mathbf{E}^{y}$. To simulate circular incident polarization, the calculated field maps $\mathbf{E}^{x}$ and $\mathbf{E}^{y}$ were combined with complex coefficients, namely, $\mathbf{E}^{+}=$ $(1 / \sqrt{2})\left(\mathbf{E}^{x}+i \mathbf{E}^{y}\right)$ and $\mathbf{E}^{-}=(1 / \sqrt{2})\left(\mathbf{E}^{x}-i \mathbf{E}^{y}\right)$ for left and right circular polarization, respectively.

[1] C. Manzo and M. F. Garcia-Parajo, A Review of Progress in Single Particle Tracking: From Methods to Biophysical Insights., Rep. Prog. Phys. 78, 124601 (2015).

[2] H. Deschout, F. C. Zanacchi, M. Mlodzianoski, A. Diaspro, J. Bewersdorf, S. T. Hess, and K. Braeckmans Precisely and Accurately Localizing Single Emitters in Fluorescence Microscopy, Nat. Methods 11, 253 (2014).

[3] W. P. Dempsey, S. E. Fraser, and P. Pantazis, SHG Nanoprobes: Advancing Harmonic Imaging in Biology, BioEssays 34, 351 (2012).

[4] A. Crut, P. Maioli, N. D. Fatti, and F. Vallée, Optical Absorption and Scattering Spectroscopies of Single Nano-Objects, Chem. Soc. Rev. 43, 3921 (2014).

[5] C. Macias-Romer, M. E. P. Didier, V. Zubkovs, L. Delannoy, F. Dutto, A. Radenovic, and S. Roke, Probing Rotational and Translational Diffusion of Nanodoublers in Living Cells on Microsecond Time Scales, Nano Lett. 14, 2552 (2014).

[6] T. Fujiwara, K. Ritchie, H. Murakoshi, K. Jacobson, and A. Kusumi, Phospholipids Undergo Hop Diffusion in Compartmentalized Cell Membrane, J. Cell Biol. 157, 1071 (2002).

[7] H. Ueno, S. Nishikawa, R. Iino, K. V. Tabata, S. Sakakihara, T. Yanagida, and H. Noji, Simple Dark-Field Microscopy with Nanometer Spatial Precision and Microsecond Temporal Resolution, Biophys. J. 98, 2014 (2010).

[8] Y. Gu, X. Di, W. Sun, G. Wang, and N. Fang, ThreeDimensional Super-Localization and Tracking of Single Gold Nanoparticles in Cells, Anal. Chem. 84, 4111 (2012).

[9] J. Ortega-Arroyo and P. Kukura, Interferometric Scattering Microscopy (ISCAT): New Frontiers in Ultrafast and Ultrasensitive Optical Microscopy, Phys. Chem. Chem. Phys. 14, 15625 (2012).

[10] D. Lasne, G. A. Blab, S. Berciaud, M. Heine, L. Groc, D. Choquet, L. Cognet, and B. Lounis, Single Nanoparticle Photothermal Tracking (SNAPT) of 5-nm Gold Beads in Live Cells, Biophys. J. 91, 4598 (2006).

[11] C. Pache, N. L. Bocchio, A. Bouwens, M. Villiger, C. Berclaz, J. Goulley, M. I. Gibson, C. Santschi, and T. Lasser, Fast Three-Dimensional Imaging of Gold Nanoparticles in Living Cells with Photothermal Optical Lock-in Optical Coherence Microscopy, Opt. Express 20, 21385 (2012).

[12] D. Lasne, G. A. Blab, F. De Giorgi, F. Ichas, B. Lounis, and L. Cognet, Label-Free Optical Imaging of Mitochondria in Live Cells, Opt. Express 15, 14184 (2007).

[13] Y. Gu, J. W. Ha, A. E. Augspurger, K. Chen, S. Zhu, and N. Fang, Single Particle Orientation and Rotational 
Tracking (SPORT) in Biophysical Studies, Nanoscale 5, 10753 (2013).

[14] S. M. Anthony and Y. Yu, Tracking Single Particle Rotation: Probing Dynamics in Four Dimensions, Anal. Methods 7, 7020 (2015).

[15] Y. Peng, B. Xiong, L. Peng, H. Li, Y. He, and E. S. Yeung, Recent Advances in Optical Imaging with Anisotropic Plasmonic Nanoparticles, Anal. Chem. 87, 200 (2015).

[16] J. Olson, S. Dominguez-Medina, A. Hoggard, L.-Y. Wang, W.-S. Chang, and S. Link, Optical Characterization of Single Plasmonic Nanoparticles, Chem. Soc. Rev. 44, 40 (2015).

[17] A. Boivin and E. Wolf, Electromagnetic Field in the Neighborhood of the Focus of a Coherent Beam, Phys. Rev. 138, B1561 (1965).

[18] P. Török, P. Higdon, and T. Wilson, On the General Properties of Polarised Light Conventional and Confocal Microscopes, Opt. Commun. 148, 300 (1998).

[19] O. Schubert, J. Becker, L. Carbone, Y. Khalavka, T. Provalska, I. Zins, and C. Sönnichsen, Mapping the Polarization Pattern of Plasmon Modes Reveals Nanoparticle Symmetry, Nano Lett. 8, 2345 (2008).

[20] L. M. Payne, W. Langbein, and P. Borri, PolarizationResolved Extinction and Scattering Cross-Section of Individual Gold Nanoparticles Measured by Wide-Field Microscopy on a Large Ensemble, Appl. Phys. Lett. 102, 131107 (2013).

[21] L. Payne, G. Zoriniants, F. Masia, K. P. Arkill, P. Verkade, D. Rowles, W. Langbein, and P. Borri, Optical MicroSpectroscopy of Single Metallic Nanoparticles: Quantitative Extinction and Transient Resonant Four-Wave Mixing, Faraday Discuss. 184, 305 (2015).

[22] O. L. Muskens, G. Bachelier, N. D. Fatti, and F. Vallée, Quantitative Absorption Spectroscopy of a Single Gold Nanorod, J. Phys. Chem. C 112, 8917 (2008).

[23] W.-S. Chang, J. W. Ha, L. S. Slaughter, and S. Link, Plasmonic Nanorod Absorbers as Orientation Sensors, Proc. Natl. Acad. Sci. U.S.A. 107, 2781 (2010).

[24] N. Gao, Y. Chen, L. Li, Z. Guan, T. Zhao, N. Zhou, P. Yuan, S. Q. Yao, and Q.-H. Xu, Shape-Dependent Two-Photon Photoluminescence of Single Gold Nanoparticles, J. Phys. Chem. C 118, 13904 (2014).

[25] N. K. Balla, C. Rendón-Barraza, L. M. Hoang, P. Karpinski, E. Bermúdez-Ureña, and S. Brasselet, Polarized Nonlinear Nanoscopy of Metal Nanostructures, ACS Photonics 4, 292 (2017).
[26] F. Masia, W. Langbein, P. Watson, and P. Borri, Resonant Four-Wave Mixing of Gold Nanoparticles for ThreeDimensional Cell Microscopy, Opt. Lett. 34, 1816 (2009).

[27] F. Masia, W. Langbein, and P. Borri, Measurement of the Dynamics of Plasmons Inside Individual Gold Nanoparticles Using a Femtosecond Phase-Resolved Microscope, Phys. Rev. B 85, 235403 (2012).

[28] C. F. Bohren and D. R. Huffman, Absorption and Scattering of Light by Small Particles (Wiley-VCH, New York, 1998).

[29] See Supplemental Material at http://link.aps.org/ supplemental/10.1103/PhysRevX.7.041022 for additional information (text and figures) on calculations and experiments.

[30] B. T. Miles, E. C. Robinson, E. M. H. P. van Dijk, I. D. Lindsay, N. F. van Hulst, and H. Gersen, Sensitivity of Interferometric Cross-Polarization Microscopy for Nanoparticle Detection in the Near-Infrared, ACS Photonics 2, 1705 (2015).

[31] H. Katz-Boon, C. J. Rossouw, M. Weyland, A. M. Funston, P. Mulvaney, and J. Etheridge, Three-Dimensional Morphology and Crystallography of Gold Nanorods, Nano Lett. 11, 273 (2011).

[32] A. B. Taylor, A. M. Siddiquee, and J. W. M. Chon, Below Melting Point Photothermal Reshaping of Single Gold Nanorods Driven by Surface Diffusion, ACS Nano 8, 12071 (2014).

[33] A. Yildiz et al., Myosin V Walks Hand-Over-Hand: Single Fluorophore Imaging with 1.5- $\mathrm{nm}$ Localization, Science 300, 2061 (2003).

[34] M. J. Nasse and J. C. Woehl, Realistic Modeling of the Illumination Point Spread Function in Confocal Scanning Optical Microscopy, J. Opt. Soc. Am. A 27, 295 (2010).

[35] J. J. M. Braat, P. Dirksen, A. J. E. M. Janssen, S. van Haver, and A. S. van de Nes, Extended Nijboer-Zernike Approach to Aberration and Birefringence Retrieval in a HighNumerical-Aperture Optical System, J. Opt. Soc. Am. A 22, 2635 (2005).

[36] C. J. R. Sheppard and P. Török, Efficient Calculation of Electromagnetic Diffraction in Optical Systems Using a Multipole Expansion, J. Mod. Opt. 44, 803 (1997).

[37] S. S. Sherif, M. R. Foreman, and P. Török, Eigenfunction Expansion of the Electric Fields in the Focal Region of a High Numerical Aperture Focusing System, Opt. Express 16, 3397 (2008).

[38] http://doi.org/10.17035/d.2017.0031438581. 